\title{
Competencias del cuidador familiar para cuidar a su familiar que recibe quimioterapia
}

\author{
Competence of the family caregiver to care for a family member receiving chemotherapy
}

Competências do cuidador de família para cuidar o seu familiar que recebe quimioterapia

\author{
Hanier Fajardo-Rocha* \\ Amparo Montalvo-Prieto**
}

\section{Resumen}

Introducción: La presencia de una enfermedad aguda, crónica o terminal constituye una crisis familiar. El diagnóstico de cáncer afecta no solo al individuo, sino también al ser humano que pertenece a una sociedad, a una familia impactada por este diagnóstico. Objetivo: Asociar las condiciones sociodemográficas y el nivel de competencias que poseen los cuidadores familiares para el cuidado en el hogar de pacientes en quimioterapia. Materiales y métodos: Estudio descriptivo correlacional, aplicado a 123 personas mayores de 18 años, dedicadas al cuidado en el hogar de su familiar con cáncer entre la segunda y sexta sesión de quimioterapia, dispuestos a participar en el estudio. Se utilizaron los instrumentos de caracterización de los cuidadores familiares de América Latina ${ }^{\circledR}$, APGAR familiar y Competencia para el cuidado - cuidador, CUIDAR, versión abreviada. Para el análisis, se realizaron distintas regresiones logísticas multivariadas para cada dimensión así como para la competencia global. Para determinar los factores asociados se estimaron los odds ratio junto con sus respectivos intervalos de confianza. Resultados: Las participantes del estudio fueron mujeres, pertenecientes al estrato socioeconómico uno y con formación secundaria, que se perciben con una alta competencia para cuidar en el hogar. Se encontró asociación entre las dimensiones de cuidar y algunas de las características sociodemográficas. La competencia global cuidar se asoció a las condiciones de ser mujer, dedicar más de ocho horas diarias al cuidado y pertenecer a una familia funcional. Conclusiones: Las condiciones sociodemográficas y de funcionalidad familiar se asocian a las dimensiones que conforman las competencias cuidar y la competencia global cuidar.

Palabras clave: Cuidado, cáncer, quimioterapia, habilidades de cuidado, competencia

\section{Abstract}

Introduction: The presence of an acute, chronic or terminal disease constitutes a family crisis. The diagnosis of cancer not only affects the individual but the human being
Autor de correspondencia

* Enfermera, egresada Universidad de Cartagena, Estudiante especialización en Enfermería cardiovascular, Universidad Nacional de Colombia. Enfermera asistencial, Clínica Palermo. Correo: hannifr12@ hotmail.com. Orcid: https://orcid.org/00000002-1572-8112 Bogotá, Colombia.

** Enfermera, Magister en Enfermería- Docente, Profesor Titular, Facultad de Enfermería-Universidad de Cartagena. Correo: amontalvop1@unicartagena.edu.co. Orcid: https;// Orcid.org/0000-0002-7925-8674. Cartagena, Colombia.
Este es un artículo bajo la licencia CC BY (https://creativecommons.org/ licenses/by/4.0/) @) (1)

\section{Para citar este artículo/ To reference this article / Para citar este artigo/}

Fajardo Rocha H, Montalvo Prieto A. Competencias del cuidador familiar para cuidar a su familiar que recibe quimioterapia. Rev. cienc. cuidad. 2019; 16(2): 95-107 
ISSN-PRINT

1794-9831

E-ISSN 2322-7028

Vol. 16 No. 2

May - Ago 2019

Cúcuta, Colombia

that belongs to a society, to a family that is impacted by this diagnosis. Objective: Associate the sociodemographic conditions and the level of competence that family caregivers have for the home care of patients in chemotherapy. Materials and methods: Correlational descriptive study applied to 123 people older than 18, dedicated to the home care of a family member with cancer between the second and sixth chemotherapy session, willing to participate in the study. The instruments of characterization of Latin American family caregivers ${ }^{\circledR}$ used were, family APGAR and Competence for Care- caregiver, CUIDAR, abbreviated version. For the analysis, different multi-varied logistic regressions were performed for each dimension as well as for global competence. To determine the associated factors, odds ratio were estimated along with their respective confidence interval. Results: The participants of the study were women that belong to the social stratus one and have secondary education, who perceive themselves as highly competent for the care of a family member at home. A relationship was found between the dimensions of care and some of the sociodemographic characteristics. The global care competence was associated to the conditions of being a woman, dedicating more than eight hours a day to the care of the family member and belonging to a functional family. Conclusions: The sociodemographic and functionality conditions of the family are associated to the dimensions that constitute the care competences and the global care competences.

Keywords: care, cancer, chemotherapy, care skills, competence.

\section{Resumo}

Introdução: A presença de uma doença aguda, crónica ou terminal constitui uma crise na família. O diagnóstico de câncer afeta não só quem a padece, também ao ser humano que faz parte da sociedade e a sua família impactada por esse diagnóstico. Objetivo: Associar as condições sociais e demográficas com o nível de competência que possuem os cuidadores de família para o cuidado domiciliar de pacientes em quimioterapia. Materiais e métodos: Estudo descritivo de correlação, feito em familiares cuidadores domiciliares de parentes com câncer entre a segunda e sexta sessão de quimioterapia, maiores de 18 anos e que aceitaram a participação voluntária no estudo. Utilizaramse a escala de caracterização dos cuidadores de família de América Latina®, APGAR familiar e a escala CUIDAR versão abreviada. Para a análise, realizaram-se diversas regressões logísticas multivariadas para cada dimensão, assim como para a competência global. Para determinar os fatores associados estimou-se o Odds ratio com seus respetivos intervalos de confiança. Resultados: As participantes do estudo foram mulheres do nível socioeconômico um, com o ensino médio e perceberam-se com alta competência para o cuidado domiciliar. Encontrou-se associação entre as dimensões da escala CUIDAR e algumas das características sociais e demográficas. A competência global para o cuidado associou-se às condições de ser mulher, dedicar mais de oito horas diárias aos cuidados e pertencer a uma família funcional. Conclusões: As condições sociais, demográficas e de funcionalidade familiar estiveram associadas com as dimensões que conformam a competência e a competência global para cuidar.

Palavras-chave: Cuidado, câncer, quimioterapia, competência, cuidador familiar.

\section{Introducción}

La presencia de una enfermedad aguda, crónica o terminal en uno de los miembros de la familia se constituye en una crisis que la perturba. El cáncer está incluido dentro de las enfermedades crónicas y su diagnóstico afecta a todo el sistema familiar (1). Ante un diagnóstico sobre cáncer no solo se tiene un cuerpo enfermo, sino también a un ser humano que pertenece a una sociedad, a una familia, la cual se va a ver impactada por este diagnóstico (2). Las estadísticas mundiales acerca del cáncer prevén que el número de casos en los próximos 20 años aumentarán aproximadamente en un 70\%; se estima que en Estados Unidos para el 2016 la incidencia fue de 1.685.210, en el 2015 en España se diagnosticaron más de 220.000 personas (3), en Colombia el cáncer afectara aproximadamente a 80.000 personas (4), es decir, que un gran número de familias se enfrentaran a este tipo de padecimiento y a las consecuencias del tratamiento de la enfermedad.

Por otra parte, el diagnóstico de cáncer impacta a los pacientes y a su familia, ocasionando sentimientos de 
depresión, ansiedad y miedo, emociones que se consideran normales ante la experiencia de una situación que pone en riesgo la vida (5). Esta gran confusión en que se encuentra el paciente puede afectar sus relaciones personales, por la preocupación relacionada con los síntomas, el tratamiento y la muerte. El apoyo psicológico puede resultar de gran ayuda y su propósito es que las personas aprendan a sobrellevar los cambios (físicos, emocionales y de estilo de vida) y el tratamiento médico que puede ser doloroso y traumático (6).

Se debe señalar, que la familia, los amigos y los profesionales de la salud se constituyen en un recurso de apoyo en el plano emocional o instrumental porque proporcionan un mayor nivel de satisfacción a los pacientes (7). Los requerimientos económicos y emocionales en esta enfermedad son altos, pues tratar con la patología requiere de materiales (medicamentos de alto costo, exámenes diagnósticos complejos, tratamiento multidisciplinario, hospitalizaciones) y de profesionales de la salud que puedan atender las dimensiones humanas del paciente, que se ven afectadas desde el momento del diagnóstico, durante el tratamiento y en la rehabilitación (8). La persona con cáncer suele presentar cambios a nivel emocional como pena y tristeza que pueden estar acompañadas de ansiedad, angustia, desesperanza; pero también puede sentir rabia, ira y enojo, además de la sensación de estar solo en esto. Es por todo lo anterior que estos pacientes requieren no sólo de un abordaje médico y biológico, sino que necesitan de un cuidado integral, lo que implica un trabajo en equipo, con una mirada biopsicosocial donde se ayude a entender cómo afecta el cáncer al paciente y su familia (9).

Como medida para descongestionar los centros hospitalarios gran parte de la atención del enfermo se ha trasladado al hogar, las estadías en los hospitales son cortas, hay tratamientos manejables que no requieren estar en el hospital durante la noche o estar bajo supervisión continúa de personal de salud, por lo cual el familiar a cargo del paciente se vuelve parte de este equipo (10). En su estudio Puerto (11) confirma que las necesidades físicas y emocionales del enfermo son asumidas principalmente por las mujeres hijas, hermanas, esposa, quienes se constituyen en los cuidadores familiares, puesto que se perciben como el mayor soporte social de su núcleo familiar.

En todo caso, las funciones del cuidador familiar para con el paciente con cáncer radican en la asistencia a los malestares físicos y emocionales originados a partir de las quimioterapias. Jaman y Rivera (12) expresan en su estudio, que las pacientes manifestaban a nivel corporal periodos de malestar general, dolores en el cuerpo, náuseas, cansancio, falta de apetito, cambios de temperatura corporal, pérdida de vitalidad, fuerza y energía, por lo que dependía de sus familiares la realización de las actividades de cuidados básicos. Además, las pacientes presentaban cambios emocionales como ansiedad, miedos e incertidumbre por el tratamiento, las complicaciones y la posibilidad de reaparición de la enfermedad. Por su parte, los profesionales de la salud manifestaron que cuentan con el tiempo justo para brindar atención e informar, pero muy poco para profundizar en aspectos específicos del paciente; igualmente, mencionan que si el tiempo es casi nulo para atender al enfermo, es mucho menor el que le pueden dedicar a los familiares.

Según un estudio realizado por Carreño y Arias (13) se encontró un nivel medio de competencia para cuidar en el hogar, condición que debe ser intervenida; en cambio, cuando el cuidador está preparado para cuidar en el hogar, puede anticiparse a las necesidades de cuidado de su familiar enfermo y disminuye la presencia de sobrecarga. También se resalta que los cuidadores familiares de niños con cáncer requieren mejorar su competencia para cuidar en el hogar, con especial énfasis en sus condiciones personales, en aspectos relacionados con el cuidado diario y de relación social.

Dentro de esa perspectiva, es de suma importancia adquirir las aptitudes idóneas para realizar una acción o intervenir en asuntos determinados con el paciente; es decir, que se deben poseer las competencias necesarias para desempeñarse en una actividad en particular. Carrillo et al., (14) definen las competencias para el cuidado en el hogar como aquellas capacidades, habilidades y preparación que adquieren los usuarios o el cuidador familiar para ejercer la labor de cuidar.

Es importante brindar acompañamiento a la familia durante el proceso de la enfermedad y hacer seguimiento de la calidad del cuidado en el hogar, lo que significa un compromiso dentro de la prestación de servicios de salud por parte de las IPS; esta actitud le reportará beneficios, disminuyendo los reingresos hospitalarios y la demanda en el servicio de urgencias. De igual manera, las tasas de infecciones domiciliarias serían menores, con menos gastos en in- 
ISSN-PRINT

1794-9831

E-ISSN 2322-7028

Vol. 16 No. 2

May - Ago 2019

Cúcuta, Colombia sumos y medicamentos por eventos asociados a los cuidados del paciente. El acompañamiento continúo durante todo el proceso de enfermedad al paciente y la familia debe ser un compromiso por el cual la enfermería se convierte en un soporte educacional. Por consiguiente, la profesión a su vez se beneficia, pues enriquece su quehacer obteniendo nuevos conocimientos para la implementación del cuidado, evalúa la atención que se está brindando y con base a los resultados modificar los aspectos negativos de los servicios prestados.

Es necesario precisar, que el cuidador familiar principal, asume la responsabilidad de cuidar al paciente por largas jornadas, a veces las 24 horas del día; en ese sentido, algunos deben renunciar a su trabajo a pesar del aumento de los gastos por el tratamiento. Más aún, no disfrutan de actividades recreativas por su dedicación al enfermo y muchas veces descuidan su propia salud por estar pendientes del familiar al que están cuidando, lo que puede hacer que la persona dedicada al cuidado sea víctima de un estado de sobrecarga o cansancio crónico (15). El apoyo del personal de salud podría ser significativamente importante para el cuidador, pues contaría con un soporte social que le ayudaría a disminuir los niveles de sobrecarga; igualmente, podría desempeñar mejor su rol al adquirir mayores conocimientos sobre el tratamiento de la enfermedad, lo que le permitiría sentirse más útil y capacitado para desempeñar el rol que está ejecutando y así asimilar rápidamente la situación de la enfermedad. Para el sujeto de cuidado contar con el apoyo emocional de su familiar y además saber que está debidamente entrenado, le genera mayor seguridad al estar en el hogar; factores que facilitan el fortalecimiento de los lazos familiares y el paciente al tener menores dificultades en su tratamiento podrá regresar prontamente a sus actividades habituales.

Sin embargo, en el ambiente clínico se observa el desplazamiento del familiar durante el proceso de tratamiento de la enfermedad, mientras tanto el acompañante se ubica en la sala de espera estando ajeno a la atención que se le está brindando al enfermo. Terminada la sesión se lo entregan transfiriéndole desde ese momento la responsabilidad de cuidarlo, de modo que no se tiene en cuenta la magnitud del compromiso que significa para el cuidador hacerse cargo del paciente. Es decir, que no hay ninguna información ni educación por parte del personal de salud sobre el manejo del enfermo en casa; en suma, es como si el compromiso del equipo de salud de velar por el bienestar del enfermo se acabara al momento de enviarlo a su domicilio.

Es también relevante tener en cuenta que al entrevistar a los familiares de pacientes con cáncer en dos instituciones de salud, se percibió como queja principal la falta de educación ofrecida al cuidador para el tratamiento en el hogar; a este respecto, algunos expresan que ningún miembro del equipo de salud se acercó a decirles lo que podría pasar en casa. Llama la atención el hecho de que al salir los pacientes de las sesiones de quimioterapia y ser enviados al domicilio, son recibidos por los familiares, quienes no han recibido ningún asesoramiento para controlar los efectos adversos del tratamiento, haciéndose evidente la exclusión del familiar en el proceso terapéutico. Es por ese motivo, que los cuidadores han aprendido durante el proceso de enfermedad por ensayo y error, por experiencias de otras personas e inclusive a través de internet. Algunos familiares manifestaron temor por llevar al enfermo a casa, pues desconocían los cuidados que debían brindarle, muchos referían que el querer proteger al familiar les servía de impulso para saber cómo actuar.

\section{Objetivos}

\section{Objetivo General}

Se estableció como propósito del presente estudio, asociar las condiciones sociodemográficas y el nivel de competencias que poseen los cuidadores familiares para el cuidado en el hogar de pacientes en quimioterapia.

\section{Objetivos específicos}

- Describir el perfil sociodemográfico del cuidador familiar principal de pacientes en tratamiento de quimioterapia.

- Evaluar la funcionalidad familiar a través del APGAR en las familias de pacientes en tratamiento de quimioterapia.

- Identificar las habilidades y destrezas para las labores del cuidado por parte del cuidador familiar de pacientes en quimioterapia.

- Reconocer el grado de bienestar del cuidador familiar de pacientes en quimioterapia. 


\section{Materiales y Métodos}

Estudio cuantitativo de tipo descriptivo correlacional, realizado a 123 cuidadores familiares principales de pacientes con cáncer, que se encuentren entre la segunda y sexta sesión de quimioterapia y que asisten a dos instituciones de salud en Cartagena. Se estableció como criterio de inclusión ser mayor de 18 años, dedicado al cuidado en el hogar de un familiar que recibe quimioterapia. Los datos de los pacientes se obtuvieron de una base de datos proporcionada por las instituciones y a partir del cumplimiento de los criterios de inclusión de los pacientes se escogieron a los cuidadores familiares principales. La información fue recolectada teniendo en cuenta a los participantes en la institución o en su domicilio. Se utilizaron: el instrumento de caracterización de los cuidadores familiares de América Latina ${ }^{\circledR}(16)$, el APGAR familiar (17) y el instrumento competencia para para el cuidado - cuidador, versión abreviada (18).

El APGAR familiar identifica la funcionalidad de la familia en un momento determinado, conformado por 5 preguntas con opciones de respuesta de 0 a 4 , donde 0 corresponde a nunca y 4 a siempre. Cuenta con un índice de correlación que oscila entre 0.71 y 0.83 y un alfa de Cronbach de 0.84 encontrado en estudios de validez y fiabilidad (19). El instrumento competencia del cuidado cuidador (CUIDAR) versión abreviada, consta de 20 ítems divididos en seis dimensiones: Conocimiento, Unicidad (condiciones personales), Instrumental y procedimental, Disfrutar (bienestar), Anticipación (predictiva) y Relación social e interacción, con opciones de respuesta en una escala tipo Likert que va de 0 a 3 , donde 0 es casi nunca o nunca y 3 casi siempre o siempre. Para evaluar la competencia del cuidado, el instrumento tiene tres puntos de corte, el nivel bajo con un puntaje de 0-36, el puntaje de 37-48 corresponde a medio y alto y un puntaje de 49-60 (20). El instrumento cuenta con una validez de 0.96 (20); para este estudio el alfa de Cronbach encontrado fue de 0,72 .

A los participantes del estudio se les dio a conocer el objetivo y alcances del estudio; igualmente, se les garantizó la confidencialidad y una que vez que accedieron a participar, firmaron el consentimiento informado; a continuación se recolectó la información contenida en los tres instrumentos. La información fue recolectada por una de las investigadoras y dos auxiliares de investigación previamente entrenadas.
Los datos se incorporaron en una matriz en Microsoft Excel 2010; luego se interpretaron empleando el paquete estadístico SPSS versión 22.0. Se utilizó estadística descriptiva para las variables cuantitativas. Mediante el análisis multivariado se realizaron distintas regresiones logísticas multivariadas tanto para cada dimensión del instrumento como para el instrumento en su totalidad. Para determinar los factores asociados se estimaron los odds ratio junto con sus respectivos intervalos de confianza.

Durante el desarrollo de la investigación se tuvo en cuenta lo establecido en la resolución 008430 de 1994 del Ministerio de Salud de Colombia, la cual establece las normas científicas, técnicas y administrativas para realizar investigaciones en salud (21). El estudio contó con el aval de las instituciones de salud y del Comité de ética de la Universidad de Cartagena (FECEI-0092016). Por otra parte, el uso del instrumento sobre competencia del cuidado cuidador (CUIDAR) versión abreviada, fue autorizado por la líder del grupo "Cuidado de enfermería al paciente crónico" de la Universidad Nacional de Colombia.

\section{Resultados}

\section{Características sociodemográficas de los cuidado- res.}

Participaron un total de 123 cuidadores familiares principales de pacientes diagnosticados con cáncer que asistían entre la segunda y sexta sesión de quimioterapia. El 79,67\% (98) fueron mujeres con edades entre los 26 y 59 años de edad y el 73,97\% (91) con más de 60 años; el 13,01\% (16) procedía del área rural; el 52,03\% (64) y el 57,72\% (71) con pareja estable; y un $45 \%$ (39) pertenecía al estrato uno. Se encontraban dedicadas principalmente a las actividades del hogar el 48,78\% (60), al trabajo independiente el $24,39 \%$ (30) y a un trabajo estable el $18,70 \%$ (23). La población de estudio contó con una formación académica de secundaria en un 58,54\% (72), un 17,07\% (21) tenía estudios elementales y solo el 10,57\% (13) poseía estudios universitarios. Respecto a la religiosidad, el 86,99\% (107) manifestó pertenecer a un grupo religioso y al preguntarles sobre su nivel de compromiso con sus creencias, el 41,46\% (51) lo considera alto y medio el $43,09 \%$ (53).

Con relación a la carga de cuidado se encontró que el $62,60 \%$ (77) el tiempo de diagnóstico del receptor de $\overline{\text { E-ISSN 2322-7028 }}$

Vol. 16 No. 2

May - Ago 2019

Cúcuta, Colombia 
ISSN-PRINT

1794-9831

E-ISSN 2322-7028

Vol. 16 No. 2

May - Ago 2019

Cúcuta, Colombia cuidado era menor a un año, el 21,14\% (26) de un año y el $16.26 \%$ (18) más de 2 años. En cuanto al número de horas al día que dedica al cuidado, el 55,28\% (68) expresó que todo el día, un 30,8\% (37) señaló que entre 9 a 16 horas y el 14, $62 \%$ (18) entre 1 y 8 horas. El 70,73\% (87) no es el único cuidador y el 17,07\% (21) no cuentan con ninguno tipo de apoyo para la labor de cuidar.

En cuanto al nivel de percepción de la carga de cuidado, el 69,92\% (86) manifestó tener un nivel moderado, mientras que el $8,13 \%$ (10) percibe una alta o muy alta carga de cuidado y un $21,95 \%$ (27) una baja percepción de carga. Según el tipo de relación con la persona cuidada, ser pareja o hijo (a) comparten igual porcentaje $19,51 \%$ (24), el 14,63\% (18) eran padres y el 46,34\% (59) tenían otro tipo de relación. El $78,05 \%$ (96) de los participantes afirmó no padecer enfermedades crónicas. El 68,28\% (84) cuenta con apoyo para cuidar y al 4.07\% (5) los apoyan económicamente.

\section{Funcionalidad familiar.}

Al evaluar la funcionalidad familiar, el 89,43\% (110) de las familias de los participantes del estudio conservan una muy buena funcionalidad familiar (Gráfico 1).

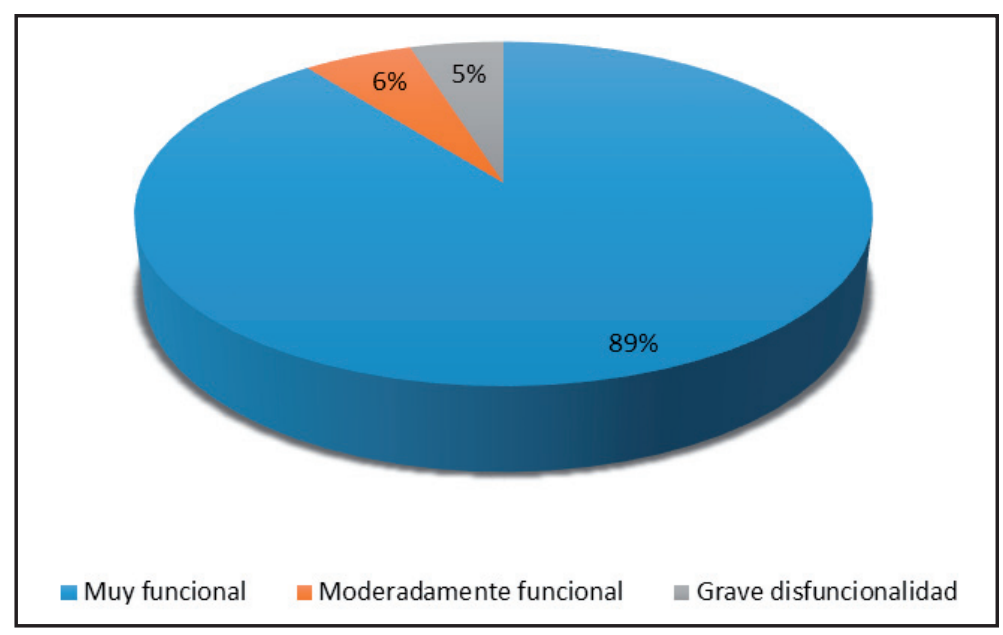

Gráfico 1. Apgar familiar de los participantes, cuidadores de pacientes que reciben quimioterapia. 2018

Fuente: Fajardo H, Montalvo A. Competencias del cuidador familiar para cuidar en el hogar a pacientes que reciben quimioterapia. Cartagena (2018).

\section{Competencia para el cuidado.}

En cuanto al nivel de competencia de los cuidadores familiares principales de las personas que se encuentran en tratamiento de quimioterapia, el 82,1\% (101) lo calificaron como alto y el $17,9 \%$ (22) consideró que posee un mediano nivel de competencias. Al revisar cada una de las dimensiones se percibe que el $27,6 \%$ (34) reconoció tener en las dimensiones conocimiento y unicidad un nivel medio de competencia, el 67,5\% (83) un alto nivel de competencias en conocimiento y en unicidad el 70,7\% (87). El 89,4\% (110) estimó que posee un alto nivel de competencia en la dimensión instrumental, la cual evalúa las actuaciones del cuidador para con la persona que cuida, siguiendo las recomendaciones del equipo de salud, organizando los recursos disponibles para el cuidado y administrando adecuadamente los medicamentos formulados; y el 87,0\% (107) evalúa la dimensión disfrutar con un alto nivel de competencia. Respecto, a la competencia de anticipación que valora en el cuidador el manejo de riesgos, de necesidades y de organización de los recursos necesarios para el cuidado, el $82,9 \%$ (102) de los participantes consideró que posee un alto nivel de competencia y el 78\% (96) le asigna igual calificación a la dimensión relación (Gráfico 2). 


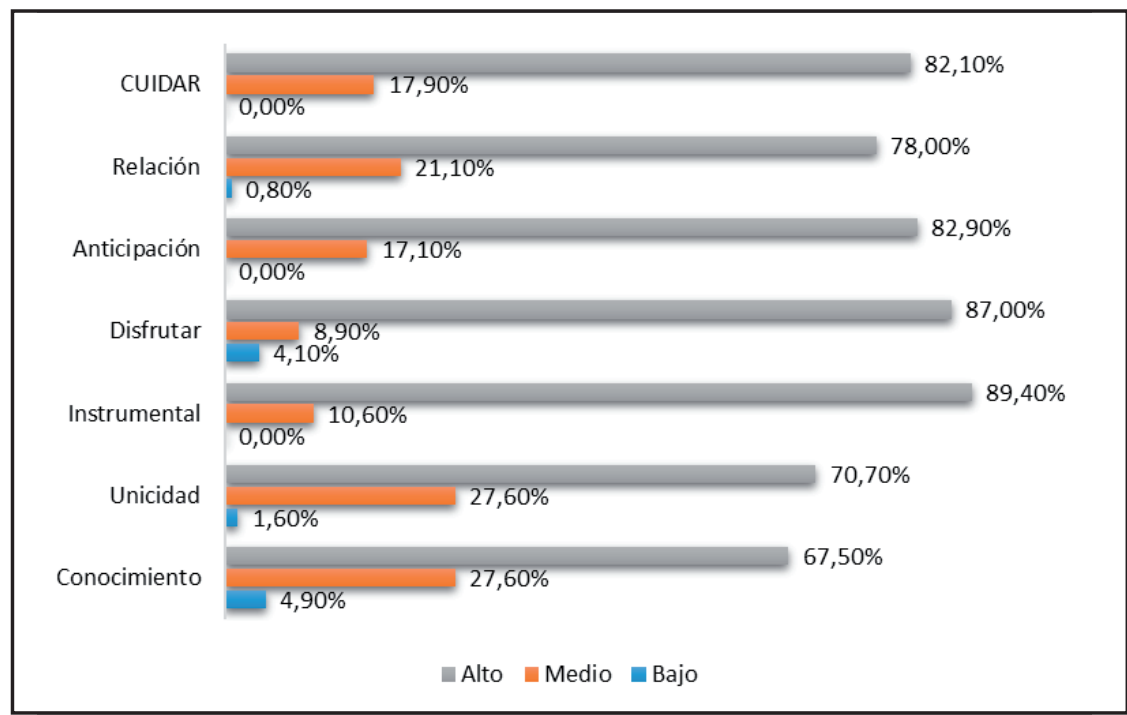

ISSN-PRINT

1794-9831

E-ISSN 2322-7028

Vol. 16 No. 2

May - Ago 2019

Cúcuta, Colombia

Gráfico 2. Competencia del cuidador familiar para cuidar en el hogar a pacientes que reciben quimioterapia (2018).

Fuente: Fajardo H, Montalvo A. Competencias del cuidador familiar para cuidar en el hogar a pacientes que reciben quimioterapia. Cartagena (2018).

$\mathrm{Al}$ asociar las condiciones del cuidador con cada una de las dimensiones que constituyen la competencia para cuidar al familiar enfermo, la dimensión conocimiento aumenta la probabilidad de estar asociada a que el cuidador trabaje. No hubo asociación entre las variables del cuidador y la dimensión unicidad, mientras que la competencia instrumental aumenta la probabilidad de asociarse a la funcionalidad familiar, debido a la mayor posibilidad de organizar los recursos. Ser único cuidador reduce la probabilidad de obtener mejores resultados en la dimensión del Disfrute -satisfacción del cuidador con su estilo de vida actual, puesto que debe tener energía para cumplir con sus responsabilidades y tiempo para realizar sus actividades personales-, mien- tras que cuando se tiene una familia funcional sucede todo lo contrario. Asimismo, el hecho de que el cuidador tenga una enfermedad, es probable que afecte de manera negativa la competencia de Anticipación -prever riesgos y organizar recursos-.

Por otra parte, al ser mujer, dedicar más de 8 horas al día al cuidado, tener más de 45 años, percibir la carga como alta y tener una familia funcional está asociado a un mejor desempeño en la competencia de Relación - contar con mayores recursos para fortalecer el vínculo de afecto-. La variable funcionalidad familiar del cuidador y ser mujer presentó una asociación positiva con la competencia global del cuidar (Tabla1). 
ISSN-PRINT

$1794-9831$

E-ISSN 2322-7028

Vol. 16 No. 2

May - Ago 2019

Cúcuta, Colombia

Tabla 1. Asociación entre las condiciones de los cuidadores y las dimensiones que constituyen las competencias para el cuidado - cuidador de pacientes que reciben quimioterapia (2018).

\begin{tabular}{|c|c|c|c|c|c|c|c|}
\hline & C & $\mathbf{U}$ & I & D & $\mathbf{A}$ & $\mathbf{R}$ & CUIDAR \\
\hline $\begin{array}{c}\text { CONDICIONES } \\
\text { CUIDADOR }\end{array}$ & OR I.C. $95 \%$ & OR I.C. 95\% & OR I.C. 95\% & OR I.C. 95\% & OR I.C. $95 \%$ & OR I.C. $95 \%$ & OR I.C. $95 \%$ \\
\hline Ser mujer & $3,6(0,9-13,7)$ & $0,9(0,2-3,1)$ & $2,7(0,5-14,9)$ & $0,5(0,1-3,9)$ & $3,2(0,6-15,8)$ & $11,2(2,3-52,1)$ & $5,9(1,1-35,5)$ \\
\hline $\begin{array}{l}\text { Ser del área } \\
\text { urbana }\end{array}$ & $1,2(0,5-3,2)$ & $0,7(0,3-1,8)$ & $1,8(0,4-7,1)$ & $1,3(0,3-5,1)$ & $2,1(0,6-6,5)$ & $1,6(0,5-5,3)$ & $1,1(0,3-4,4)$ \\
\hline $\begin{array}{l}\text { Paciente mayor } \\
\text { de } 40 \text { años }\end{array}$ & $1,1(0,4-3,2)$ & $1,9(0,7-5,2)$ & $0,5(0,1-2,6)$ & $2,1(0,4-10,2)$ & $0,3(0,2-1,2)$ & $0,9(0,2-3,5)$ & $1,3(0,3-5,8)$ \\
\hline Trabajar & $4,4(1,4-13,8)$ & $1,1(0,3-3,9)$ & $1,1(0,2-5,1)$ & $0,8(0,1-3,8)$ & $2,8(0,7-10,5)$ & $1,3(0,3-4,9)$ & $4,4(0,9-21,1)$ \\
\hline $\begin{array}{l}\text { Tener alto } \\
\text { compromiso } \\
\text { religioso }\end{array}$ & $0,8(0,3-2,1)$ & $1,5(0,5-4,1)$ & $0,5(0,1-2,5)$ & $0,8(0,1-3,5)$ & $0,9(0,2-2,9)$ & $0,5(0,1-2,1)$ & $0,4(0,1-1,9)$ \\
\hline $\begin{array}{c}\text { Ser de estrato } \\
1 \text { y } 2\end{array}$ & $1,1(0,3-3,1)$ & $0,6(0,2-1,6)$ & $1,125(0,6-2,0)$ & $0,8(0,1-4,5)$ & $0,4(0,1-1,6)$ & $0,7(0,1-2,7)$ & $0,9(0,2-4,1)$ \\
\hline $\begin{array}{l}\text { Tener un año } \\
\text { o más con la } \\
\text { enfermedad }\end{array}$ & $1,6(0,6-4,6)$ & $0,9(0,3-2,4)$ & $0,5(0,1-2,7)$ & $0,7(0,7-1,9)$ & $0,8(0,2-2,7)$ & $0,7(0,2-2,3)$ & $1,2(0,3-4,9)$ \\
\hline $\begin{array}{l}\text { Dedicar más de } \\
8 \text { horas diarias } \\
\text { al cuidado }\end{array}$ & $1,3(0,3-4,5)$ & $0,7(0,2-2,6)$ & $3,1(0,5-17,4)$ & $0,3(0,1-4,2)$ & $1,5(0,3-7,2)$ & $13,1(2,7-62,7)$ & $11,8(2,2-61,7)$ \\
\hline $\begin{array}{l}\text { Ser único } \\
\text { cuidador }\end{array}$ & $3,1(0,9-11,1)$ & $0,9(0,3-2,8)$ & $0,4(0,1-2,2)$ & $0,1(0,02-0,91)$ & $2,1(0,4-8,7)$ & $0,6(0,1-2,1)$ & $1,6(0,3-7,5)$ \\
\hline $\begin{array}{l}\text { Cuidador mayor } \\
\text { de } 45 \text { años }\end{array}$ & $0,4(0,1-1,1)$ & $1,1(0,4-2,9)$ & $0,7(0,1-3,9)$ & $0,3(0,1-1,7)$ & $0,4(0,1-1,5)$ & $6,5(1,3-33,3)$ & $2,8(0,6-13,1)$ \\
\hline $\begin{array}{c}\text { Tener una } \\
\text { enfermedad }\end{array}$ & $0,4(0,1-1,3)$ & $0,6(0,2-1,7)$ & $1,5(0,2-8,5)$ & $1,1(0,2-5,9)$ & $0,2(0,08-0,97)$ & $0,5(0,1-2,1)$ & $0,4(0,2-1,9)$ \\
\hline $\begin{array}{c}\text { Considerar la } \\
\text { carga como alta }\end{array}$ & $0,3(0,1-1,6)$ & $0,4(0,1-1,8)$ & - & $0,5(0,1-3,8)$ & $3,1(0,2-34,9)$ & $11,5(2,1-62,2)$ & $0,2(0,1-1,4)$ \\
\hline Familia funcional & $2,1(0,4-8,7)$ & $1,9(0,5-7,3)$ & $7,4(1,2-51,9)$ & $13,5(2,4-73,0)$ & $3,9(0,7-21,1)$ & $11,5(2,1-62,2)$ & $40,7(6,2-26,3)$ \\
\hline Alfa de cronbach & 0,723 & & & & & & \\
\hline
\end{tabular}

Fuente: Fajardo H, Montalvo A. Competencias del cuidador familiar para cuidar en el hogar a pacientes que reciben quimioterapia. Cartagena (2018).

\section{Discusión}

La competencia general CUIDAR se asoció de forma positiva con la variable ser mujer, dedicar más de 8 horas al cuidado y la funcionalidad familiar de los cuidadores participantes del estudio que cuidan en el hogar a personas que reciben quimioterapia. Sánchez y Carrillo (22) reportan un nivel de competencia media del cuidador familiar de la persona con cáncer en tratamiento de quimioterapia y expresan que, aunque existe el vínculo estrecho, las competencias para el cuidado se desarrollan de forma independiente (22). Es decir, que esta situación se convierte en un llamado de alerta sobre cómo asumen las instituciones de salud su responsabilidad de buscar el bienestar del paciente y del cuidador familiar durante todo el proceso de enfermedad; es por eso, que deben comprometerse a garantizar la continuidad de la atención durante la estancia hospitalaria y cuando el paciente es dado de alta. Además, el cuidador familiar debe poseer competencias para cuidar en el hogar porque éstas le brindan apoyo para el cuidado y el bienestar del paciente, así como el convertirse en un elemento importante para las instituciones de salud. En este caso es necesario que el personal que labora en las instituciones de salud, teniendo en cuenta las condiciones físicas y de salud de los pacientes, deben desarrollar un plan de egreso, que contenga un programa de fortalecimiento para el cuidador en todos los aspectos requeridos para el cuidado y para hacer frente a las diferentes terapéuticas que requiere el paciente (20). 
Llama la atención, que las participantes del estudio, fueron en su mayoría mujeres adultas, en un porcentaje apreciable mayores de 60 años, procedentes del área rural, dedicadas al hogar y con estudios de bachillerato como formación académica. Estos resultados son similares a los hallados en diferentes estudios (11, 22, 23). Por su parte, Sánchez y Carrillo (22) informan que la edad promedio de las cuidadoras fue de 45,9 con un mínimo de 18 años y un máximo de 79 años, mujeres en un 72,5\%, el bachillerato fue la formación que predominó en ellas; autores que confirman lo descrito por Fernández (24) quien expresa que la tradición cultural le ha atribuido a la mujer el valor del cuidado, como innato y necesario, constituyéndose en un elemento clave y los valores femeninos se establecen como garantes del cuidado de la sociedad (24).

Los participantes del estudio en su mayoría viven con una pareja estable, ubicadas de acuerdo a la escala socioeconómica en el estrato uno, con una alta participación religiosa. Resultados similares reportan Puerto (11) y Torres et al. (23); a su vez, Sánchez y Carrillo (22) informan en su estudio que el $66,3 \%$ tiene pareja estable, proceden de la zona urbana principalmente y practican la religión católica. También, en el presente estudio se encontró que las creencias y prácticas religiosas se constituyen en una condición relevante en las cuidadoras estudiadas. Con respecto a este aspecto, diferentes autores consideran que el apoyo religioso/espiritual se constituye en una herramienta importante para ayudar a enfrentar el cáncer y si el tema es tratado por los profesionales de salud (25) les da mayor fortaleza. Por otra parte, Puentes et al., (26) a su vez anotan que en la vivencia de la enfermedad la comprensión de la espiritualidad y la religiosidad permite un mejor manejo de la situación (26) por lo que es importante que desde su formación, el profesional de la salud aprenda a observar, escuche al otro y le ayude a buscar el apoyo espiritual y esperanza en el futuro (27).

Según este estudio los cuidadores manifiestan no padecer enfermedades crónicas, son únicos cuidadores desde hace menos de un año y a pesar de dedicar todo el día a esta labor, perciben su carga como moderada. Al respecto, Carreño y Arias (13) indican que estas condiciones son precursoras de sobrecarga para el cuidador; y según el estudio de Aldana et al., (7) los cuidadores reconocen que tienen un solo cuidador, que su familia los apoya económicamente pero sienten que representan una carga alta de cuidado (7); razón por la cual la enfermería debe intervenir para que los familiares participen en el cumplimiento del rol de cuidador. También, los cuidadores expresaron tener un buen nivel de funcionalidad familiar. En ese sentido, Acosta et al., (28) encontraron en su estudio una funcionalidad familiar moderada, con predominio en la resolución de problemas. Además, el lograr que la familia alcance una armonía a su interior, es un elemento de apoyo para el acompañamiento en las labores del cuidador y permite disminuir la carga de cuidado y de apoyo al paciente. Es por eso, que Achury et al. (29) resaltan que el profesional de enfermería debe ayudar a identificar posibles signos y síntomas de sobrecarga y crear espacios de encuentro donde se promueva el cuidado al cuidador.

El nivel de competencias global identificado en el grupo en estudio fue alto, dato similar a lo reportado por Carrillo et al. (14), para quienes este reconocimiento por parte del cuidador puede verse afectado en el contexto colombiano por los gastos financieros, el ser único cuidador y la necesidad de acompañamiento por parte del Estado en esta labor tan desgastante (30). Si se tiene en cuenta las dimensiones que constituyen la competencia CUIDAR como son: Conocimientos que comprenden el dominio de aspectos relacionados con la enfermedad, terapéutica y cuidado cotidiano; Unicidad que corresponde a las características internas y de personalidad, fueron evaluadas en un nivel alto, similar a lo reportado por Carrillo $\mathrm{G}^{14}$, pero diferentes a los resultados de Aldana et al. (7), Carreño y Arias (13) y Achury et al. (29) en los que predomina un nivel medio de competencia para estas dos dimensiones. El nivel educativo de los cuidadores limita la capacidad de comprender aspectos complejos de la información (23) por lo que las intervenciones de enfermería deben ser sencillas, claras y de manera permanente.

En lo que corresponde a las competencias instrumentales que comprenden las habilidades, -organización y uso adecuado de los elementos para el cuidado-, los resultados muestran un alto nivel; datos similares reporta Achury (29), quien anota que, a pesar de sentirse satisfechos como cuidadores, manifiestan tener poco tiempo para sus diligencias personales. En cuanto a las dimensiones, Relación e Interacción y Anticipación que se refieren a las relaciones sociales y de apoyo que tienen los cuidadores, así como el estar preparados para asumir las necesidades o la presencia de un evento inesperado respectivamente, los datos encontrados 
ISSN-PRINT

1794-9831

E-ISSN 2322-7028

Vol. 16 No. 2

May - Ago 2019

Cúcuta, Colombia reportan un alto nivel de competencia, similar a lo obtenido por Aldana et al. (7) para quienes la dimensión Relación es diferente a la de anticipación, con un nivel medio de competencia. Achury et al. (29) por su parte, informan que existen iguales resultados en los niveles medio y alto para la dimensión anticipación. La intervención a los cuidadores debe hacerse con información clara y comprensible, en donde la amabilidad en el trato, la respuesta a las inquietudes (31), permiten que la información recibida logre en el cuidador los cambios que se pretenden.

Cabe considerar, por otra parte, que al asociar las dimensiones con las variables sociodemográficas y de funcionalidad familiar, se encontró que la variable Ser cuidador y trabajar se asocia a la dimensión conocimiento; de igual forma, el ser parte de una familia con funcionalidad familiar se asocia positivamente con la dimensión instrumental y disfrute, pero el ser único cuidador disminuye la posibilidad del disfrute. El estar enfermo se asocia de forma negativa con la competencia anticipación. Del mismo modo, la dimensión relación está asociada de forma positiva con ser mujer mayor de 40 años, dedicar más de 8 horas al día al cuidado, percibir la carga del cuidado como alta y tener una familia funcional. De otra parte, Torres et al. (23) relatan que la escolaridad, el nivel socioeconómico y la edad del cuidador son factores que influyen positivamente en la habilidad del cuidador (23); también, resaltan que la enfermería debe procurar mejorar el nivel cognitivo del cuidador, fortaleciendo la formación e impulsando su autonomía. Con frecuencia, se observa en el cuidador familiar principal el estrés de cuidar un familiar en quimioterapia lo que afecta su salud (11), es por esto que un cuidador enfermo debe ser apoyado por la familia para mejorar sus condiciones al cuidar su familiar enfermo. Para concientizar a los cuidadores sobre la importancia de apoyarse en las redes de soporte social -de salud y familiar- debe hacerse a través de educación y seguimiento (32).

Es importante indicar que las participantes del estudio fueron en su mayoría mujeres, entre los 26 y 59 años de edad, pertenecientes al estrato socioeconómico uno y con formación secundaria. Igualmente, se las percibe como poseedoras de una alta competencia para CUIDAR en el hogar, traducidas en cada una de sus dimensiones
Con respecto, a las condiciones sociodemográficas y de funcionalidad familiar presentan algún tipo de asociación con las dimensiones que conforman las dimensiones de las competencias del CUIDAR.

\section{Conclusiones}

- Los cuidadores familiares principales son principalmente mujeres, con formación secundaria, en una etapa de edad productiva; proceden en su mayoría del área rural, con pareja estable y un alto compromiso religioso; igualmente, perciben como moderada la carga de cuidado del paciente que recibe quimioterapia y se encuentra entre la segunda y sexta semana de tratamiento.

- Las familias de los pacientes en tratamiento con quimioterapia entre la semana dos y sexta de su tratamiento, presentan una buena funcionalidad familiar.

- El cuidador familiar participante del presente estudio considera que posee un nivel de competencia medio en las dimensiones conocimiento y unicidad, en cuanto a su competencia para realizar seguimiento de las condiciones de salud, identificar sus necesidades y los signos de alarma; además, reconoce sus condiciones personales para cuidar.

- El estudio reportó un alto nivel para la competencia instrumental y de disfrute, debido a que el cuidador sigue las recomendaciones, organiza el cuidado y suministra el tratamiento indicado; así mismo siente satisfacción, está dispuesta, le encuentra sentido a la labor que realiza y cuenta con tiempo para cuidarse

- Los participantes del presente estudio consideraron que poseen un alto nivel de competencia para cuidar en casa a sus familiares que se encuentran en quimioterapia, entre la semana dos y sexta de su tratamiento.

\section{Agradecimientos.}

Cristina Bohórquez por su apoyo desinteresado e invaluable 
1. Crivello M. Implicancias y consecuencias de la enfermedad crónica sobre el grupo familiar. Revista Virtual de la Facultad de Psicología y Psicopedagogía de la Universidad del Salvador [Internet] 2013 [Consultado 2 de mayo de 2018]; 30: 24-36. Disponible en: http://racimo.usal.edu.ar/4585/1/12614822-1-PB.pdf

2. Rodríguez $\mathrm{Y}$, Costas R. El diagnóstico de cáncer de mamas desde una perspectiva familiar: Retos para la Psico-oncología en América Latina. Interamerican Journal of Psychology. [Internet] 2013 [Consultado 14 de mayo de 2018]; 47(1):121-130. Disponible en: http://www.redalyc.org/pdf/284/28426980014. pdf

3. Sociedad Española de Oncología Médica. El cáncer en España. [Internet]; 2015 [Consultado 17 de mayo de 2016]. Disponible en: https://seom.org/publicaciones/el-cancer-en-espanyacom

4. Organizacion Mundial de la salud (OMS). Cáncer. [Internet]. 2015 (Consultado 9 de mayo de 2016]. Disponible en: http://www.who.int/mediacentre/factsheets/fs297/es/

5. American Cancer Society. Coping with Cancer. El cáncer y los efectos emocionales. [Internet] [Consultado 10 de enero de 2018]. Disponible en: https:/www.cancer.org/es/tratamiento/tratamientos-yefectos-secundarios/efectos-secundarios-emocionales.html

6. American Psychological Association. Breast cancer: How your mind can help your body. [Internet] [Consultado 10 de enero de 2018]. Disponible en: http://www.apa.org/centrodeapoyo/cancer-de-mama.aspx

7. Aldana E, Barrera S, Rodríguez K, Gómez O, Carrillo Mabel. Competencia para el cuidado (CUIDAR) en el hogar de personas con enfermedad renal crónica en hemodiálisis. Enferm Nefrol. [Internet] 2016 [Consultado 10 de febrero de 2018] 19 (3): 265-273. Disponible en: http://scielo.isciii.es/scielo. php?script $=$ sci abstract\&pid $=$ S2254-28842016000300009

8. Gallardo K, Benavides F, Rosales R. Costos de la enfermedad crónica no trasmisible: la realidad colombiana. Rev Cienc Salud. [Internet] 2016 [Consultado 20 de febrero de 2018]; 14 (1): 103-114. Disponible en: http://revistas.urosario.edu.co/index.php/revsalud/article/view/4596

9. Robert V, Álvarez C, Valdivieso F. Psicooncología: un modelo de intervención y apoyo psicosocial. REV. MED. CLIN. CONDES. [Internet] 2013 [Consultado 10 de febrero de 2018]; 24(4): 677-684. Disponible en: https://www.sciencedirect.com/science/article/pii/S0716864013702074

10. Instituto Nacional del Cáncer (INC). Familiares a cargo de pacientes de cáncer; [Internet] 2016 [Consultado: 9 de mayo de 2016]. Disponible en: http://www.cancer.gov/espanol/cancer/sobrellevar/familia-y-amigos/familiares-a-cargo-pdq\#section/ 1

11. Puerto HM. Calidad de vida en cuidadores familiares de personas en tratamiento contra el cáncer. Rev Cuid. [Internet] 2015; [Consultado 20 de febrero de 2018] 6(2):1029-40. Disponible en: https://www. revistacuidarte.org/index.php/cuidarte/article/view/154/462

12. Jaman P, Rivera M. Vivir con cáncer: una experiencia de cambios profundos provocados por la quimioterapia. Aquichan. [Internet] 2014 [Consultado 10 de febrero de 2018]; 14(1):20-31. Disponible en: http://aquichan.unisabana.edu.co/index.php/aquichan/article/view/2394/3374

13. Carreño S, Arias M. Competencia para cuidar en el hogar y sobrecarga en el cuidador del niño con cáncer. [Internet] Gaceta Mexicana de Oncología. 2016 [Consultado 10 de febrero de 2018]; 15 (6): 336-343. Disponible en: https://www.sciencedirect.com/science/article/pii/S1665920116300864

14. Carrillo G, Barreto R, Arboleda L, Gutierrez O, Melo B, Ortiz V. Competencia para cuidar en el hogar de personas con enfermedad crónica y sus cuidadores en Colombia. Rev Fac Med. [Internet] 2015 [Consultado 10 de febrero de 2018]; 63(4): 665-675. Disponible en: http://www.scielo.org.co/pdf/ rfmun/v63n4/v63n4a12.pdf

15. Vega O, Ovallos F, Velasquez N. Sobrecarga de los cuidadores de pacientes oncológicos usuarios de la clínica cancerológica en San José de Cúcuta. Investig.Enferm. [Internet] 2012 [Consultado 10 de fe- 
ISSN-PRINT

$1794-9831$

E-ISSN 2322-7028

Vol. 16 No. 2

May - Ago 2019

Cúcuta, Colombia

brero de 2018]; 14(2): 85-95. Disponible en: http://revistas.javeriana.edu.co/index.php/imagenydesarrollo/article/view/4211

16. Chaparro L, Sánchez B, Carrillo GM. Encuesta de caracterización del cuidado de la diada cuidador familiar - persona con enfermedad crónica. Rev Cien Cuidad. [Internet] 2014 [Consultado 10 de febrero de 2018]; 11:31-45 Disponible en: http://revistas.ufps.edu.co/ojs/index.php/cienciaycuidado/article/view/196

17. Suarez M, Alcalá M. Apgar familiar: Una herramienta para detectar disfunción familiar. Rev Med La Paz. 2014 Junio; 20(1).

18. Carrillo GM, Sánchez B, Arias EM. Validation of an instrument to assess the homecare competency of the family caregiver of a person with chronic disease. Invest Educ Enferm. [Internet] 2015 [Consultado 10 de febrero de 2018]; 33(3): 449-455. Disponible en: http://www.scielo.org.co/scielo. php?script $=$ sci_arttext\&pid $=$ S0120-53072015000300008

19. Bellón J, Delgado A, Luna J, Lardelli P. Validez y fiabilidad del cuestionario de función familiar Apgar-familiar. Atención primaria. 1996; 18(6).

20. Carrillo G, Sánchez B, Vargas E. Desarrollo y pruebas psicométricas del instrumento "Cuidar"- versión corta para medir la competencia de cuidado en el hogar. Rev Univ Santander Ind Salud. [Internet] 2016 [Consultado 10 de febrero de 2018]; 48(2). Disponible en: http://revistas.uis.edu.co/index.php/ revistasaluduis/article/view/5509

21. Republica de Colombia. Ministerio de salud. Resolución N 008430 de 1993, octubre 4, por la cual se establecen las normas científicas, técnicas y administrativas para la investigación en salud [Internet]. Santa Fe de Bogotá: El Ministerio; 1993 [consultado 29 de octubre de 2016]. Disponible en: https://www.minsalud.gov.co/sites/rid/Lists/BibliotecaDigital/RIDE/DE/DIJ/RESOLUCION8430-DE-1993.PDF

22. Sánchez LM, Carrillo GM. Competencia para el cuidado en el hogar diada persona con cáncer en quimioterapia-cuidador familiar. Psicooncología [Internet] 2017 [Consultado 10 de febrero de 2018]; 14: 137-48. Disponible en: http://revistas.ucm.es/index.php/PSIC/article/view/55817

23. Torres X, Carreño S, Chaparro L. Factores que influencian la habilidad y sobrecarga del cuidador familiar del enfermo crónico. Rev Univ Ind Santander Salud. [Internet] 2017 [Consultado 10 de febrero de 2018]; 49(2): 330-338. Disponible en: http://revistas.uis.edu.co/index.php/revistasaluduis/article/ view/6359

24. Fernandez M. El origen de la mujer cuidadora: apuntes para el análisis hermenéutico de los primeros testimonios. Index Enferm (Gran). [Internet] 2016 [Consultado 10 de febrero de 2018]; 25(1-2):93-97. Disponible en: http://scielo.isciii.es/scielo.php?script=sci_arttext\&pid=S1132-12962016000100021

25. Mesquita AC, Cássia E, Valcanti C, Alves D, Gerhke R, Campos de Carvalho E. La utilización del enfrentamiento religioso/espiritual por pacientes con cáncer en tratamiento quimioterápico. Rev. LatinoAm. Enfermagem. [Internet] 2013 [Consultado 2 de mayo de 2018]; 21(2). Disponible en: http://www. scielo.br/pdf/rlae/v21n2/es_0104-1169-rlae-21-02-0539.pdf

26. Puentes YM, Urrego SC, Sánchez R. Espiritualidad, religiosidad y enfermedad: una mirada desde mujeres con cáncer de mama. Avances en Psicología Latinoamericana. [Internet] 2015 [Consultado 2 de mayo de 2018]; 33(3): 481-495. Disponible en: https://revistas.urosario.edu.co/ xm1/799/79940724009/79940724009_visor_jats.pdf

27. Espíndula JA, Valle E, Bello A. Religión y espiritualidad: una perspectiva de profesionales de la salud. Rev. Latino-Am. Enfermagem [Internet]. 2010 [Consultado 12 de mayo de 2018]; 18(6): Disponible en: http://www.scielo.br/pdf/rlae/v18n6/es_25.pdf

28. Acosta E, López C, Martínez ME, Zapata R. Funcionalidad familiar y estrategias de afrontamiento en pacientes con cáncer de mama. Horizonte sanitario [Internet] 2017 [Consultado 12 de mayo de 2018]; 16 (2). Disponible en: http://www.scielo.org.mx/scielo.php?script=sci_arttext\&pid $=\mathrm{S} 2007-74592017000200139$ 
29. Achury DM, Restrepo A, Torres NM, Buitrago AL, Neira NX, Devia P. Competencia de los cuidadores familiares para cuidar a los pacientes con falla cardíaca. Rev Cuid. [Internet] 2017 [Consultado 12 de mayo de 2018]; 8(3): 1721-32. Disponible en: https://www.revistacuidarte.org/index.php/cuidarte/ article/view/407

30. Mayorga J, Montoya LA, Montoya I, Sánchez B, Sandoval NM. Metodología para analizar la carga financiera del cuidado familiar de una persona con enfermedad crónica. Rev.cienc.biomed. [Internet] 2015 [Consultado 12 de mayo de 2018]; 6(1): 96-106. Disponible en: http://revistas.unicartagena.edu. co/index.php/cienciasbiomedicas/article/view/1267/1177

31. Fernández LE, Conde S, Fernández JS. Grado de satisfacción de los cuidadores principales de pacientes seguidos por los equipos de cuidados paliativos. Semergen. [Internet] 2016 [Consultado 12 de mayo de 2018]; 42 (7): 476-481. Disponible en: http://www.elsevier.es/es-revista-medicina-familiasemergen-40-articulo-grado-satisfaccion-los-cuidadores-principales-S1138359316000915

32. Carrillo G, Sánchez B, Gómez O, Carreno S, Chaparro L. Metodología de implementación del "plan de egreso hospitalario" para personas con enfermedad crónica en Colombia. Revista Med 25 años. [Internet] 2017 [Consultado 14 de mayo de 2018]; 25 (2): 55-62. Disponible en: https://revistas.unimilitar.edu.co/index.php/rmed/article/view/3079 